TRANSACTIONS OF THE

AMERICAN MATHEMATICAL SOCIETY

Volume 184, October 1973

\title{
UNIVERSAL GENERATORS FOR VARIETIES OF NUCLEAR SPACES
}

BY

\section{B. ROSENBERGER}

ABSTRACT. It is shown that a product of several copies of $\Lambda\left(\beta^{\phi}\right)$ is a universal $\phi$-nuclear space if the power series space $\Lambda\left(\beta^{\phi}\right)$ with $\beta_{k}^{\phi}=$ $-\log \left(\phi^{-1}(1 / \sqrt{k+1})\right), k \in\{0,1,2, \ldots\}$, is $\phi$-nuclear; here $\phi=[0, \infty) \rightarrow[0, \infty)$ is a continuous, strictly increasing subadditive function with $\phi(0)=0$. In case $\Lambda\left(\beta^{\phi}\right)$ is not $\phi$-nuclear the sequence space $\Lambda\left(l_{\phi}^{+}\right)$is a $\phi$-nuclear space with the property that every $\phi$-nuclear space is isomorphic to a subspace of a product of $\Lambda\left(l_{\phi}^{+}\right)$if $\lim \sup _{t \rightarrow 0}(\phi(t))^{-1} \phi(\sqrt{t})<\infty$.

1. Introduction. In [3] Diestel, Morris, and Saxon studied varieties of locally convex Hausdorff spaces, i.e. classes of locally convex Hausdorff spaces closed under the formation of subspaces, separated quotients, arbitrary products, and isomorphic images. They showed that each variety $\mathcal{O}$ generated by a single locally convex space has a universal generator, i.e. $\overparen{C}$ contains a locally convex space $E$ such that each locally convex space $F \in \mathcal{O}$ is isomorphic to a subspace of a product of copies of $E$. For the variety $\pi$ of nuclear spaces Komura and Komura [7] proved that the Fréchet space $s$ of rapidly decreasing sequences is a universal generator. In [11] Ramanujan proved that the strong dual $[\Lambda(\alpha)]_{b}^{\prime}$ of the power series space $\Lambda(\alpha)$ where $\sup _{n} a_{2 n} a_{n}^{-1}<\infty$ for $a=\left\{a_{n}\right\}_{n \in N_{0}}$ is a universal generator for the variety $\pi_{\Lambda(a)}$ of $\Lambda(a)$-nuclear spaces. As a special case he obtained Martineau's result [8] that the strong dual $(s)_{b}^{\prime}$ of the Fréchet space $s$ is a universal generator for the variety $\pi_{s}$ of $s$-nuclear spaces.

In this paper we describe concrete universal generators for the variety $\pi_{\phi}$ of $\phi$-nuclear spaces where $\phi:[0, \infty) \rightarrow[0, \infty)$ is a continuous, strictly increasing, subadditive function with $\phi(0)=0$ (cf. [13]). Generalizing a result of Komura and Kömura [7] we obtain the power series space $\Lambda\left(\beta^{\phi}\right)$ with $\beta_{k}^{\phi}=-\log \left(\phi^{-1}(1 / \sqrt{k+1})\right)$, $k \in \mathbf{N}_{0}$, as a universal generator for the variety $\pi_{\phi}$, if $\Lambda\left(\beta^{\phi}\right)$ is $\phi$-nuclear; for $\phi_{p}(t)=t^{p}, 0<p \leq 1$, we get the power series space $s$.

For functions $\phi$ with the additional property that $\lim \sup _{t \rightarrow 0}(\phi(t))^{-1} \phi(\sqrt{t})$ $<\infty$ - this especially implies that the power series space $\Lambda\left(\beta^{\phi}\right)$ is not $\phi$-nuclearwe obtain a result similar to the case of $\Lambda(\alpha)$-nuclear spaces; it is shown that in this case the sequence space $\Lambda\left(l_{\phi}^{+}\right)$is a universal generator for $\pi_{\phi}$.

Received by the editors December 18, 1972 and, in revised form, March 26, 1973.

AMS (MOS) subject classifications (1970). Primary 46A05, Secondary 46A45, 46A99.

Key words and phrases. Nuclear spaces, sequence spaces, variety, universal generator. 
2. Notation and terminology.

2.1. For terminology not explained here see [5] or [10]. Let $E$ always be a locally convex Hausdorff space; we denote by $\mathcal{U}(E)$ a fundamental system of neighbourhoods of 0 (neighbourhood base at 0 ) consisting of closed, absolutely convex, and absorbing neighbourhoods of 0 . Given $U \in \mathcal{U}(E)$, let $p_{U}$ be the corresponding seminorm defined by $p_{U}(x)=\inf \{a>0: U \in a U\}$. We shall consider the linear space $E(U):=E / \operatorname{ker} p_{U}$ normed by $p_{U}$, and the completion $\widehat{E(U)}$ of $E(U)$. Given two neighbourhoods of $0, U, V \in \mathcal{U}(E)$ with $V \subset a U$ for some $a>0$ (written $V<U$ ), the canonical map $E(V, U)$ is the linear map of $E(V)$ onto $E(U)$ which associates the element $x(U)$ to the equivalence class $x(V) ; E(V, U)$ is continuous, and $E(V, U)$ is its unique continuous extension to the completions.

2.2. For two subsets $A, B \subset E$ with $A \subset a B$ for some $a>0$ (written $A<B$ ) and for a subspace $E_{n} \subset E$ with $\operatorname{dim} E_{n} \leq n$, we define

$$
\delta(A, B ; E):=\inf \left\{\delta>0: A \subset \delta B+E_{n}\right\} .
$$

The n-diameter of $A$ with respect to $B$ (in the sense of Kolmogorov) is then defined by

$$
\delta_{n}(A, B):=\inf \left\{\delta\left(A, B ; E_{n}\right): E_{n} \subset E, \operatorname{dim} E_{n} \leq n\right\}
$$

for $n \in \mathbf{N}_{0}:=\{0,1,2, \cdots\}$. It is immediately clear that for $U, V \in \mathcal{U}(E)$ we have $\delta_{n}(V, U)=\delta_{n}(V(U), U(U)), n \in \mathbf{N}_{0}$, where $V(U)$ (resp. $U(U)$ ) is the set of all equivalence classes $v(U)$ (resp. $u(U)$ ) with $v \in V$ (resp. $u \in U$ ) in $E(U)$.

2.3. Let $\mathfrak{B}(E)$ be the set of all closed, absolutely convex, and bounded subsets of $E$. For $B \in \mathfrak{B}(E)$, we consider the normed linear space $E(B):=\{x \in E$ : $x \in a B$ for some $a>0\}$ with the norm $p_{B}$ defined by $p_{B}(x):=\inf \{a>0: x \in a B\}$. Since for $A \in \mathfrak{B}(E)$ and $U \in \mathcal{U}(E)$ we have $A<U$, the canonical map $E(A, U)$ of $E(A)$ into $E(U)$ is defined by $E(A, U)_{x}=x(U)$ for $x \in E(A)$. For two subsets $A, B \in \mathfrak{B}(E)$ with $A<B$, the canonical map $E(A, B)$ of $E(A)$ into $E(B)$ is defined by $E(A, B) x=x$ for $x \in E(A) . E(A, B)$ again is linear and continuous. The strong dual $[E(U)]_{b}^{0}$ of the Banach space $E(U)$ can be identified with the Banach space $E^{\prime}\left(U^{0}\right)$, where $U^{0}$ is the polar of $U \in \mathcal{U}(E)$.

2.4. Let $P$ be a set of scalar sequences $\rho=\left\{\rho_{n}\right\}_{n \in N_{0}}$ such that (i) $\rho_{n} \geq 0$ for each $n \in \mathbf{N}_{0}$, (ii) for each $n \in \mathbf{N}_{0}$ there exists a sequence $\rho \in P$ such that $\rho_{n}>0$, and (iii) for each sequence $\rho \in P$ and each $\sigma \in P$ there exists a sequence $r \in P$ such that $\sup \left\{\sigma_{n}, \rho_{n}\right\} \leq \tau_{n}$ for each $n \in N_{0}$. We define the sequence space $\Lambda(P)$ by

$$
\Lambda(P):=\left\{\xi=\left\{\xi_{k}\right\}_{k \in \mathrm{N}_{0}}: p_{\rho}(\xi)=\sum_{k=0}^{\infty}\left|\xi_{k}\right| \rho_{k}<\infty \text { for each } \rho \in P\right\} .
$$


The seminorms $p_{\rho}, \rho \in P$, define a Hausdorff, locally convex topology on $\Lambda(P)$, $\Lambda(P)$ is complete.

A special class of sequence spaces which we shall consider will be the power series spaces (of infinite type). Let $P$ be a set of sequences $\left\{\rho^{a_{k}}\right\}_{k \in N_{0}}$ with $0<\rho<\infty$, where $a=\left\{a_{k}\right\}_{k \in N_{0}}$ is a sequence such that $0 \leq a_{0} \leq a_{1} \leq a_{2} \leq \cdots$. The sequence space $\Lambda(P)$ obtained from this set $P$ is called the power series space $\Lambda(\alpha) . \Lambda(\alpha)$ is provided with the topology generated by the seminorms $p_{m}(\xi)=\Sigma_{k=0}^{\infty}\left|\xi_{k}\right| m{ }^{a_{k}}, m \in \mathbf{N}=\{1,2, \ldots\}$. The case $a_{k}=\log (k+1), k \in \mathbf{N}_{0}$, gives the space $s$ of rapidly decreasing sequences.

2.5. Following Diestel, Morris, Saxon [3], we call a nonempty class $\mathcal{O}$ of locally convex spaces a variety if it is closed under the operations of taking subspaces, quotient spaces, (arbitrary) cartesian products and isomorphic images.

Let $C$ be a class of locally convex spaces and let $\mathcal{Q}(C)$ be the intersection of all varieties containing $C$. Then $O(C)$ is said to be the variety generated by C. If $C$ consists of a single locally convex space $E$, then $\bigcirc(C)=\mathcal{O}(E)$ is said to be singly generated. If a variety $\mathcal{O}$ contains a locally convex space $E$ such that each locally convex space in $\mathcal{O}$ is isomorphic to a subspace of a product of copies of $E$, then $E$ is called a universal generator for $\mathcal{O}$.

2.6. We shall consider the set $\Phi$ of all continuous, strictly increasing, subadditive functions $\phi:[0, \infty) \rightarrow[0, \infty)$ satisfying the condition $\phi(0)=0$. For any such function $\phi \in \Phi$ we shall denote by $l_{\phi}$ the collection of all real sequences $\left\{\xi_{k}\right\}_{k \in \mathrm{N}_{0}}$ that are summed by $\phi$, i.e. satisfying the condition

$$
\sum_{k=0}^{\infty} \phi\left(\left|\xi_{k}\right|\right)<\infty
$$

By a result of Bessaga, Pelczyński, Rolewicz [1] each function $\phi \in \underset{\sim}{\Phi}$ is equivalent to a concave function $\tilde{\widetilde{\phi}} \in \Phi$ (i.e. equivalent to a function $\tilde{\phi} \in \Phi$ satisfying $1 / 2(\tilde{\phi}(s)+\widetilde{\phi}(t)) \leq \widetilde{\phi}((s+t) / 2)$ for $s, t \in[0, \infty))$. Since $\phi \in \Phi$ the function $a \phi$ belongs to $\Phi$ for any nonnegative real scalar $\alpha$, we may always assume that $\phi \in \Phi$ is concave and is satisfying the condition $\phi(1)=1$. It is clear that $l_{\phi}$ depends only on the behavior of $\phi \in \Phi$ in an arbitrarily small interval $[0, \epsilon)$.

Examples.

$$
\phi_{p}(t)=t^{p} \text { for } t \in[0, \infty), 0<p \leq 1
$$

$$
\phi_{\log }(t)= \begin{cases}0 & \text { for } t=0, \\ -a / \log t & \text { for } t \in\left(0, t_{0}\right], t_{0} \text { sufficiently small, } \\ b t+c & \text { for } t \geq t_{0},\end{cases}
$$

where $a, b, c \in \mathbf{R}^{+}$are chosen in such a way that $\phi_{\text {log }} \in C^{1}[0, \infty)$ and $\phi_{\text {log }}(1)=1$ 
(iii)

$$
\psi^{p}(t)= \begin{cases}0 & \text { for } t=0 \\ a_{p} \exp \left(-(\log 1 / t)^{p}\right) & \text { for } t \in\left(0, t_{0}\right] \\ b_{p} t+c_{p} & \text { for } t \geq t_{0}\end{cases}
$$

with $0<p \leq 1, t_{0}$ sufficiently small, and where $a_{p^{\prime}} b_{p^{\prime}} c_{p} \in \mathbf{R}$ are again chosen in such a way that $\psi^{p} \in C^{1}[0, \infty)$ and $\psi^{p}(1)=1$. For $p=1$, we get $\psi^{1}(t)=\phi_{1}(t)=t$.

2.7. In [13] a locally convex space $E$ is called $\phi$-nuclear, $\phi \in \Phi$; if a fundamental system $\mathrm{U}(E)$ has the property that for each $U \in \mathcal{U}(E)$ there exists a $V \in \mathcal{U}(E)$ such that $V<U$ and $\left\{\delta_{n}(V, U)\right\}_{n \in \mathrm{N}_{0}} \in l_{\phi^{*}}$. For $\phi_{p} \in \Phi, 0<p \leq 1, E$ is nuclear $[10,9.4 .1]$ It is easily seen that each $\phi$-nuclear space is nuclear.

2.8. Let $X$ and $Y$ be two normed linear spaces and $T$ be a continuous linear map on $X$ into $Y . T$ is called $\phi$-nuclear [12], if it admits the representation

$$
T x=\sum_{k=1}^{\infty} \lambda_{k} a_{k}(x) y_{k} \text { for each } x \in X
$$

where $\left\{\lambda_{k}\right\}_{k \in \mathrm{N}} \in l_{\phi}, a_{k} \in X^{\prime},\left\|a_{k}\right\| \leq 1$, and $y_{k} \in Y$ with $\left\|y_{k}\right\| \leq 1$. For $\phi=\phi_{1}, T$ is nuclear. For Hilbert spaces $X, Y$ a nuclear operator can be written in the form

$$
T x=\sum_{n=0}^{\infty} \delta_{n}(T(U), v)\left(x, e_{n}\right) f_{n}
$$

where $U$ (resp. $V$ ) is the unit ball of $X$ (resp. $Y$ ) and $\left\{e_{n}\right\}_{n \in \mathrm{N}_{0}}$ (resp. $\left\{f_{n}\right\}_{n} \in \mathrm{N}_{0}$ ) is a complete $O N$ system in $X$ (resp. $Y$ ) $[10,8.3 .2]$.

3. Preliminary results. In this section we present some results which will be used later. The proof of the following lemma can be found in $[9,1.13]$.

Lemma 3.1. A necessary and sufficient condition that convergence of the series $\Sigma_{k=0}^{\infty} \phi\left(\left|\xi_{k}\right|\right), \phi \in \Phi$, implies convergence of the series $\Sigma_{k=0}^{\infty} \psi\left(\left|\xi_{k}\right|\right)$, $\psi \in \Phi$, (i.e. $l_{\phi}\left(l_{\psi}\right.$ ), is the existence of positive numbers $M$ and $\epsilon$ such that $\psi(t) \leq M \phi(t)$ for $0 \leq t<\epsilon$.

A more compact way of characterizing the relation $l_{\phi} \subset l_{\psi}$ is to say $\lim \sup _{t \rightarrow 0} \psi(t) / \phi(t)<\infty$.

We also need the following generalization of the Hölder inequality.

Lemma 3.2. Let $\phi \in \Phi$. Then we have

$$
\sum_{k=1}^{\infty} \phi\left(\xi_{k} \eta_{k}\right) \leq C(\xi, \eta)\left(\sum_{k=1}^{\infty} \phi\left(\xi_{k}^{p}\right)\right)^{1 / p}\left(\sum_{k=1}^{\infty} \phi\left(\eta_{k}^{q}\right)\right)^{1 / q}
$$

$\left(p^{-1}+q^{-1}=1,1<p<\infty\right)$ for sequences $\left\{\xi_{k}\right\}_{k \in \mathrm{N}_{0}},\left\{\eta_{k}\right\}_{k \in \mathrm{N}_{0}}$ of nonnegative numbers such that the series on the right side converge. 
Proof. By the well-known inequality $c_{k} d_{k} \leq p^{-1} c_{k}^{p}+q^{-1} d_{k}^{q}, k \in \mathbf{N}$, valid for $c_{k}, d_{k}>0$ and $p^{-1}+q^{-1}=1,1<p<\infty$, and with

$$
c_{k}=\xi_{k}\left(\sum_{k=1}^{\infty} \phi\left(\xi_{k}^{p}\right)\right)^{-1 / p}, d_{k}=\eta_{k}\left(\sum_{k=1}^{\infty} \phi\left(\eta_{k}^{q}\right)\right)^{-1 / q}, k \in \mathbf{N}^{.}
$$

(here we may assume that the series do not vanish), we find that

$$
\begin{aligned}
\phi\left(c_{k} d_{k}\right) & \leq \phi\left(\xi_{k}^{p} p^{-1}\left(\sum_{k=1}^{\infty} \phi\left(\xi_{k}^{p}\right)\right)^{-1}\right)+\phi\left(\eta_{k}^{q} q^{-1}\left(\sum_{k=1}^{\infty} \phi\left(\eta_{k}^{q}\right)\right)^{-1}\right) \\
& \leq \max \left\{1 ; p^{-1}\left(\sum_{k=1}^{\infty} \phi\left(\xi_{k}^{p}\right)\right)^{-1}\right\} \phi\left(\xi_{k}^{p}\right)+\max \left\{1 ; q^{-1}\left(\sum_{k=1}^{\infty} \phi\left(\eta_{k}^{q}\right)\right)^{-1}\right\} \phi\left(\eta_{k}^{q}\right)
\end{aligned}
$$

for $k \in \mathbf{N}$. On the other hand we obtain the following inequality:

$$
\begin{aligned}
\phi\left(c_{k} d_{k}\right) & =\phi\left(\left(\sum_{k=1}^{\infty} \phi\left(\xi_{k}^{p}\right)\right)^{-1 / p}\left(\sum_{k=1}^{\infty} \phi\left(\eta_{k}^{q}\right)\right)^{-1 / q} \xi_{k} \eta_{k}\right) \\
& \geq M_{0}^{-1}\left(\sum_{k=1}^{\infty} \phi\left(\xi_{k}^{p}\right)\right)^{-1 / p}\left(\sum_{k=1}^{\infty} \phi\left(\eta_{k}^{q}\right)\right)^{-1 / q} \phi\left(\xi_{k} \eta_{k}\right)
\end{aligned}
$$

where $M_{0}:=\min \left\{C \geq 1: C\left(\sum_{k=1}^{\infty} \phi\left(\xi_{k}^{p}\right)\right)^{1 / p}\left(\sum_{k=1}^{\infty} \phi\left(\eta_{k}^{q}\right)\right)^{1 / q} \geq 1\right\}$.

For $n \in \mathbf{N}$ we therefore have

$$
\begin{aligned}
\sum_{k=1}^{\infty} \phi\left(\xi_{k} \eta_{k}\right) \leq & M_{0}\left(\sum_{k=1}^{\infty} \phi\left(\xi_{k}^{p}\right)\right)^{1 / p}\left(\sum_{k=1}^{\infty} \phi\left(\eta_{k}^{q}\right)\right)^{1 / q} \\
\cdot & {\left[\max \left\{1 ; p^{-1}\left(\sum_{k=1}^{\infty} \phi\left(\xi_{k}^{p}\right)\right)^{-1}\right\} \sum_{k=1}^{n} \phi\left(\xi_{k}^{p}\right)\right.} \\
& \left.+\max \left\{1 ; q^{-1}\left(\sum_{k=1}^{\infty} \phi\left(\eta_{k}^{q}\right)\right)^{-1}\right\} \sum_{k=1}^{n} \phi\left(\eta_{k}^{q}\right)\right]
\end{aligned}
$$

such that for $n \rightarrow \infty$

$$
\sum_{k=1}^{\infty} \phi\left(\xi_{k} \eta_{k}\right) \leq M\left(\sum_{k=1}^{\infty} \phi\left(\xi_{k}^{p}\right)\right)^{1 / p}\left(\sum_{k=1}^{\infty} \phi\left(\eta_{k}^{q}\right)\right)^{1 / q} .
$$

As an immediate consequence we have the following

Theorem 3.3. If $\left\{\xi_{k}^{p}\right\}_{k \in N} \in l_{\phi}$ and $\left\{\eta_{k}^{q}\right\}_{k \in N} \in l_{\phi}, \xi_{k}, \eta_{k} \geq 0$, the sequence $\left\{\left(\xi_{k} \eta_{k}\right)^{r}\right\}_{k \in N}$ belongs to $l_{\phi}$ where $p^{-1}+q^{-1}=r^{-1}, p>0, q>0$, and we bave

$$
\left(\sum_{k=1}^{\infty} \phi\left(\xi_{k} \eta_{k}^{r}\right)\right)^{1 / r} \leq C(\xi, \eta)\left(\sum_{k=1}^{\infty} \phi\left(\xi_{k}^{p}\right)\right)^{1 / p}\left(\sum_{k=1}^{\infty} \phi\left(\eta_{k}^{q}\right)\right)^{1 / q} .
$$

From this the orem we get a new characterization of $\phi$-nuclear spaces.

Corollary. For $\phi \in \Phi E$ is $\phi$-nuclear if and only if for each positive number $p$ and eacb $U \in \mathrm{U}(E)$ there exists a neigbbourbood $V \in \mathcal{U}(E)$ with $V<U$ such that $\left\{\delta_{n}(V, U)^{p}\right\}_{n \in \mathrm{N}_{0}} \in l_{\phi^{\bullet}}$ 
Proof. Given $p>0$, choose $m \in \mathbf{N}$ such that $1 \leq m p$. Since $E$ is $\phi$-nuclear, let $U=U_{0}, U_{1}, \cdots, U_{m}$ be chosen such that $U_{k+1}<U_{k}$ and $\left\{\delta_{n}\left(U_{k+1}, U_{k}\right)\right\}_{n} \in \mathbb{N}_{0}$ $\epsilon l_{\phi}, k \in\{0,1, \cdots, m-1\}$. By the composition formula $\delta_{n}(A, B) \delta_{m}(B, C) \geq$ $\delta_{n+m}(A, C)$ for sets $A, B, C \subset E$ with $A<B<C$, we have

$$
\phi\left(\left(\delta_{m n}\left(U_{m}, U\right)\right)^{1 / m}\right) \leq \phi\left(\prod_{k=0}^{m-1}\left(\delta_{n}\left(U_{k+1}, U_{k}\right)\right)^{1 / m}\right) .
$$

Hence by Theorem 3.3,

$$
\left(\sum_{n=0}^{\infty} \phi\left(\left(\delta_{m n}\left(U_{m}, U\right)\right)^{1 / m}\right)\right)^{m} \leq C \prod_{k=0}^{m-1} \sum_{n=0}^{\infty} \phi\left(\delta_{n}\left(U_{k+1}, U_{k}\right)\right)<\infty .
$$

Thus, since the sequence of $n$-diameters is decreasing,

$$
\begin{aligned}
\sum_{n=0}^{\infty} \phi\left(\delta_{n}\left(U_{m}, U\right)^{1 / m}\right) & =\sum_{n=0}^{\infty} \sum_{i=0}^{m-1} \phi\left(\delta_{m n+i}\left(U_{m}, U\right)^{1 / m}\right) \\
& \leq m \sum_{n=0}^{\infty} \phi\left(\delta_{m n}\left(U_{m}, U\right)^{1 / m}\right)<\infty,
\end{aligned}
$$

so that $\left\{\delta_{n}(V, U)^{p}\right\}_{n} \in \mathbb{N}_{0} \in l_{\phi}$ with $V:=U_{m}$.

The following characterization of $\phi$-nuclear spaces can be found in $[13,1.13]$.

Theorem 3.4. The following assertions are equivalent:

(i) $E$ is $\phi$-nuclear, $\phi \in \Phi$.

(ii) There exists a fundamental system $\mathrm{U}(E)$ of neigbbourboods in $E$ which bas the property that for each $U \in U(E)$ there exists a $V \in \mathcal{U}(E)$ with $V<U$ such that the canonical map $\widehat{E(V, U)}$ of $E(V)$ into $E(U)$ is $\phi$-nuclear.

(iii) There exists a fundamental system $U(E)$ which bas the property that for each $U \in \mathrm{U}(E)$ there exists a $V \in \mathcal{U}(E)$ with $V<U$ such that the canonical map of $E^{\prime}\left(U^{0}\right)$ into $E^{\prime}\left(V^{0}\right)$ is $\phi$-nuclear.

(iv) Eacb fundamental system $\mathrm{U}(E)$ bas properties (ii) and (iii).

Remark. Since each $\phi$-nuclear space $E, \phi \in \Phi$, is nuclear, the fundamental system $\mathcal{U}_{H}(E)$ can be chosen in such a way that $E(U)$ and $E^{\prime}\left(U^{0}\right), U \in \mathcal{U}_{H}(E)$, are Hilbert spaces. The canonical map of $E^{\prime}\left(U^{0}\right)$ into $E^{\prime}\left(V^{0}\right)$ therefore admits the representation

$$
\begin{aligned}
E(V, U)^{\prime}: E^{\prime}\left(U^{0}\right) & \rightarrow E^{\prime}\left(V^{0}\right) \\
x & \rightarrow \sum_{n=0}^{\infty} \delta_{n}(V, U)\left(x, e_{n}\right) f_{n}
\end{aligned}
$$

where $\left\{e_{n}\right\}_{n} \in \mathrm{N}_{0}$ (resp. $\left\{f_{n}\right\}_{n} \in \mathrm{N}_{0}$ ) is a complete ON system in $E^{\prime}\left(U^{0}\right)$ (resp. $\left.E^{\prime}\left(V^{0}\right)\right)$ and $\left\{\delta_{n}(V, U)\right\}_{n} \in \mathrm{N}_{0} \in l_{\phi^{\circ}}$ 
4. Universal generators for the variety $\varkappa_{\phi}$. In [13] it is shown that for each $\phi \in \Phi$ the class $r_{\phi}$ of all $\phi$-nuclear spaces is a variety. We therefore have

Theorem 4.1. For each $\phi \in \Phi$ the variety $\varkappa_{\phi}$ bas a universal generator.

Proof. Since the variety $\pi$ of nuclear spaces is singly generated and since a subvariety of a singly generated variety is again singly generated [3, Corollary 2.8], the variety $r_{\phi}$ is singly generated for each $\phi \in \Phi$. $r_{\phi}$ therefore has a universal generator [3, The orem 2.11].

A deep result of Kōmura and Kömura [7] says that the Fréchet space $s$ of rapidly decreasing sequences is a universal generator for the variety $\boldsymbol{h}$ of nuclear spaces. Modifying the proof of this theorem of Komura and Komura we show that the power series space $\Lambda\left(\beta^{\phi}\right)$ with $\beta_{k}^{\phi}=-\log \left(\phi^{-1}(1 / \sqrt{k+1})\right), k \in \mathbf{N}_{0}$, is a universal generator for the variety $\gamma_{\phi}$ if $\Lambda\left(\beta^{\phi}\right)$ is $\phi$-nuclear.

First we need a lemma which improves a result given in [14, Lemma 1.5].

Lemma 4.2. For $\phi \in \Phi$, a locally convex space $E$ is $\phi$-nuclear if and only if for eacb $p>0$ and for each $U \in \mathcal{U}(E)$ there exists a neighbourbood $V_{p} \in \mathcal{U}(E)$ with $V_{p}<U$ such that

$$
\sum_{k=0}^{\infty} \phi\left(\left(\phi^{-1}\left(\frac{1}{k+1}\right)\right)^{-p} \delta_{k}\left(V_{p}, U\right)\right)<\infty .
$$

Proof. Since $\phi(1)=1$, we have $\left(\phi^{-1}(1 /(k+1))\right)^{p} \leq 1$ for each $k \in \mathbf{N}_{0}$ and for $p>0$. Thus

$$
\sum_{k=0}^{\infty} \phi\left(\delta_{k}\left(V_{p}, U\right)\right) \leq \sum_{k=0}^{\infty} \phi\left(\left(\phi^{-1}\left(\frac{1}{k+1}\right)\right)^{-p} \delta_{k}\left(V_{p}, U\right)\right)<\infty,
$$

i.e., $E$ is $\phi$-nuclear.

If $E$ is $\phi$-nuclear there exists for each neighbourhood $U \in \mathcal{U}(E)$ a neighbourhood $W<U$ such that $\left\{\delta_{k}(W, U)\right\}_{k \in \mathrm{N}_{0}} \in l_{\phi}$. For $p>0$ we choose a neighbourhood $V_{p} \in U(E)$ with $V_{p}<W$ such that

$$
\sum_{k=0}^{\infty} \phi\left(\left(\delta_{k}\left(V_{p}, W\right)\right)^{1 / p}\right) \leq 2^{-1}
$$

(cf. Corollary 3.3). Using the fact that the sequence $\left\{\delta_{k}\left(V_{p}, W\right)\right\}_{k \in \mathrm{N}_{0}}$ is decreasing, we obtain $(k+1) \phi\left(\left(\delta_{k}\left(V_{p}, W\right)\right)^{1 / p}\right) \leq 2^{-1}$ so that $\delta_{k}\left(V_{p}, W\right) \leq\left(\phi^{-1}(1 /(2 k+2))\right)^{p}$ for $k \in \mathbf{N}_{0}$. The desired result then follows from the inequality

$$
\begin{gathered}
\sum_{k=0}^{\infty} \phi\left(\left(\phi^{-1}\left(\frac{1}{k+1}\right)\right)^{-p} \delta_{k}\left(V_{p}, U\right)\right) \leq 2 \sum_{k=0}^{\infty} \phi\left(\left(\phi^{-1}\left(\frac{1}{2 k+2}\right)\right)^{-p} \delta_{2 k}\left(V_{p}, U\right)\right) \\
\leq 2 \sum_{k=0}^{\infty} \phi\left(\left(\phi^{-1}\left(\frac{1}{2 k+2}\right)\right)^{-p} \delta_{k}\left(V_{p}, W\right) \delta_{k}(W, U)\right) \leq 2 \sum_{k=0}^{\infty} \phi\left(\delta_{k}(W, U)\right)<\infty .
\end{gathered}
$$


Definition 4.3. For a locally convex space $E$ we denote by $\Delta(E)$, the diametral dimension of $E$, the set of all nonnegative sequences $\delta=\left\{\delta_{k}\right\}_{k} \in N_{0}$ such that for each $U \in \mathcal{U}(E)$ there exists a $V \in \mathcal{U}(E)$ with $V<U$ and $\lim _{k \rightarrow \infty} \delta_{k}^{-1} \delta_{k}(V, U)=0$ (cf. [2]).

We then have

Corollary 4.4. For a $\phi$-nuclear space, $\phi \in \Phi$, and for each $p>0$ the sequence $\left\{\left(\phi^{-1}(1 /(k+1))\right)^{p}\right\}_{k \in N_{0}}$ belongs to the diametral dimension $\Delta(E)$.

Remark. The condition given in this corollary is not sufficient. Consider the sequence $a_{n}:=(n+2) \log (n+2), n \in \mathbf{N}_{0}$; the corresponding power series space $\Lambda(a)$ is not $\phi_{\text {bog }}$-nuclear [13, Korollar 3.6]. But for each $p>0$ and for each $\left\{\rho^{a_{n}}\right\}_{n \in N_{0}} \in P^{b_{g}}$ there exists a sequence $\left\{\sigma_{n}\right\}_{n \in N_{0}} \in P$ (take $\left.\sigma_{n}:=\left(e^{a p} \rho\right)^{a_{n}}, n \in N_{0}\right)$ such that

$$
\begin{aligned}
\lim _{k \rightarrow \infty}\left(\phi_{\log }^{-1}\left(\frac{1}{k+1}\right)\right)^{-p}\left(\frac{\rho}{\sigma}\right)^{a} & =\lim _{k \rightarrow \infty, k \geq k_{0}} e^{a p(k+1)} e^{-a p(k+2) \log (k+2)} \\
& \leq \lim _{k \rightarrow \infty}(k+2)^{-a p}=0 .
\end{aligned}
$$

Lemma 4.5. (i) For $\phi \in \Phi$ we bave $\Lambda\left(\beta^{\phi}\right) \subset s$.

(ii) $\Lambda\left(\beta^{\phi}\right)$ is nuclear.

(iii) $\Lambda\left(\beta^{\phi}\right)$ is $\phi$-nuclear if and only if there exists $m \in \mathbf{N}$ such that

$$
\sum_{k=0}^{\infty} \phi\left(\left(\phi^{-1}\left(\frac{1}{\sqrt{k+1}}\right)\right)^{m}\right)<\infty
$$

Proof. (i) is an immediate consequence of [11, Lemma 3$]$ and of the fact that for $\phi \in \Phi \log \left(\phi^{-1}(1 / \sqrt{k+1})\right)^{-1} \geq 2^{-1} \log (k+1), k \in \mathbf{N}_{0}$.

(ii) is a consequence of $[10,6.1 .5]$.

(iii) is a consequence of $[13$, Satz 3.6$]$.

Lemma 4.6. Let $E$ be a $\phi$-nuclear space. For each $U \in U(E)$ there exists a sequence $\left\{a_{k}\right\}_{k \in \mathrm{N}_{0}}$ of continuous linear functionals $a_{k} \in E^{\prime}$ with $U^{0} \mathrm{C}$ $\overline{a c}\left\{a_{k}, k \in N_{0}\right\}$ such that the set

$$
G_{n}:=\left\{a_{k}\left(\phi^{-1}(1 / \sqrt{k+1})\right)^{-n}, k \in \mathbf{N}_{0}\right\}
$$

is equicontinuous for eacb $n \in \mathbf{N}$.

Proof. ( $\overline{\mathrm{ac}}(A)$ denotes the closed absolutely convex hull of the set $A$.$) The$ proof is a slight modification of the result of Komura and Komura [7]. Since $E$ is $\phi$-nuclear and therefore nuclear we may choose a fundamental system $U_{H}(E)$ of neighbourhoods such that the spaces $E^{\prime}\left(U^{0}\right), U \in \mathcal{U}_{H}(E)$, are Hilbert spaces. To each $U \in \mathcal{U}_{H}(E)$ and for each $n \in \mathrm{N}$ there is a neighbourhood $V_{n} \in \mathcal{U}_{H}(E)$ with 
$V_{n}<U$ such that by Theorem 3.4 the canonical map $T_{n}:=E\left(V_{n}, U\right)^{\prime}$ admits the representation

$$
\begin{aligned}
T_{n}: E^{\prime}\left(U^{0}\right) & \rightarrow E^{\prime}\left(V_{n}^{0}\right) \\
x & \rightarrow \sum_{k=0}^{\infty} \delta_{k}\left(V_{n}, U\right)\left(x, e_{k}^{n}\right) f_{k}^{n}
\end{aligned}
$$

where $\left\{e_{k}^{n}\right\}_{k \in \mathrm{N}_{0}}$ (resp. $\left\{f_{k}^{n}\right\}_{k \in \mathrm{N}_{0}}$ is an ON system in $E^{\prime}\left(U^{0}\right)$ (resp. $E^{\prime}\left(V_{n}^{0}\right)$ ) and

$$
\sum_{k=0}^{\infty} \phi\left(\left(\phi^{-1}\left(\frac{1}{k+1}\right)\right)^{-n} \delta_{k}\left(V_{n}, U\right)\right)=: \phi\left(\rho_{n}\right)<\infty
$$

(Lemma 4.2). Thus $\left(\phi^{-1}(1 /(k+1))\right)^{-n} \delta_{k}\left(V_{n}, U\right) \leq \rho_{n}$ for each $k \in \mathbf{N}_{0}$. From $\left(x, e_{k}^{n}\right)=0$ it follows $x=0$, therefore the ON systems $\left\{e_{k}^{n}\right\}_{k} \in N_{0}, n \in N$, are complete in $E^{\prime}\left(U^{0}\right)=[E(U)]_{b}^{\prime}$. We orthonormalize the sequence $e_{0}^{0}, e_{0}^{1}, e_{1}^{1}, e_{1}^{0}, e_{0}^{2}$, $e_{1}^{2}, e_{2}^{2}, e_{2}^{1}, e_{2}^{0}, \ldots$ and obtain a complete ON system $\left\{e_{i}\right\}_{i \in \mathrm{N}_{0}}$ in $E^{\prime}\left(U^{0}\right)$ with $\left(e_{i}, e_{j}^{n}\right)=0$ for $i>\max \left\{j^{2}, n^{2}\right\}$ such that

$$
e_{i}=\sum_{j \geq i}\left(e_{i}, e_{j}^{n}\right) e_{j}^{n} \text { if } i>n^{2} .
$$

From the identity $e_{i}^{n}=\delta_{i}\left(V_{n^{\prime}} U\right) f_{i}^{n}$ it follows that

$$
\left(p_{V_{n}^{0}}\left(e_{i}\right)\right)^{2}=\sum_{j^{2} \geq i}\left|\left(e_{i}, e_{j}^{n}\right)\right|^{2} \delta_{j}\left(V_{n}, U\right)^{2} \leq \delta_{i_{0}}\left(V_{n}, U\right)^{2} \leq\left(\phi^{-1}\left(1 /\left(i_{0}+1\right)\right)\right)^{2 n} \rho_{n}^{2}
$$

where $i_{0}:=\min \{m \in N: m \geq \sqrt{i}\}$. Since $i+1 \leq\left(i_{0}+1\right)^{2}$ we have $\left(\phi^{-1}(1 / \sqrt{i+1})\right)^{-n} e_{i}$ $\epsilon \rho_{n} V_{n}$ for $i>n^{2}$. By an easy computation we get the inequality $(i+1)\left(\phi^{-1}(1 / \sqrt{i+1})\right)^{n+2} \leq\left(\phi^{-1}(1 / \sqrt{i+1})\right)^{n}$ so that $a_{i}\left(\phi^{-1}(1 / \sqrt{i+1})\right)^{-n} \epsilon$ $\rho \rho_{n+2} V_{n+2}^{0}$ for $i>n^{2}$ where $a_{i}:=\rho(i+1) e_{i}$ and $\rho=\pi / \sqrt{6}$. Thus the set $G_{n}=$ $\left\{a_{i}\left(\phi^{-1}(1 / \sqrt{i+1})\right)^{-n}: i \in \mathrm{N}_{0}\right\}$ is equicontinuous.

It remains to prove that the polar $U^{0}$ is contained in the closed absolutely convex hull of $\left\{a_{i}: i \in \mathrm{N}_{0}\right\}$. An element $a \in U^{0}$ can be written in the form

$$
a=\sum_{i=0}^{\infty}\left(a, e_{i}\right) e_{i}=\sum_{i=0}^{\infty}\left(a, e_{i}\right) \rho^{-1}(i+1)^{-1} a_{i}
$$

where $\Sigma_{i=0}^{\infty}\left|\left(a, e_{i}\right)\right|^{2} \leq 1$. With $\gamma_{i}:=\rho^{-1}(i+1)^{-1}\left(a, e_{i}\right)$ we obtain

$$
\sum_{i=0}^{\infty}\left|\gamma_{i}\right| \leq\left(\sum_{i=0}^{\infty}\left|\left(a, e_{i}\right)\right|^{2}\right)^{1 / 2} \rho^{-1}\left(\sum_{i=0}^{\infty} \frac{1}{(i+1)^{2}}\right)^{1 / 2} \leq 1 .
$$

Thus $U^{0} \subset \overline{\mathrm{ac}}\left\{a_{i}: i \in \mathrm{N}_{0}\right\}$, and the proof is complete.

Theorem 4.7. A $\phi \cdot n u c l e a r$ space $E$ is (topologically) isomorpbic to a subspace of the product $\left[\Lambda\left(\beta^{\phi}\right)\right]^{l}, \phi \in \Phi$.

Proof. Let $\left\{U_{i}\right\}_{i \in I}=\mathcal{U}(E)$ be a fundamental system of neighbourhoods of 0 . By Lemma 4.6 there exists for each neighbourhood $U_{i} \in \mathcal{U}(E)$ a sequence 
$\left\{a_{k}^{i}\right\}_{k \in \mathrm{N}_{0}}$ of continuous linear functionals $a_{k}^{i} \in E^{\prime}$ with $U_{i}^{0} \subset \overline{\mathbf{a c}}\left\{a_{k}^{i}: k \in \mathbf{N}_{0}\right\}$ such that for each $n \in \mathrm{N}$ the set

$$
G_{n}^{i}=\left\{a_{k}^{i}\left(\phi^{-1}(1 / \sqrt{k+1})\right)^{-n}: k \in \mathrm{N}_{0}\right\}
$$

is equicontinous. We define the linear map $K_{i}: E \rightarrow \Lambda\left(\beta^{\phi}\right)$ by $K_{i} x=\left\{a_{k}^{i}(x)\right\}_{k \in N_{0}}$ Since $\Lambda\left(\beta^{\phi}\right)$ is nuclear (Lemma 4.5) the topology of $\Lambda\left(\beta^{\phi}\right)$ can also be obtained by the fundamental system of neighbourhoods

$$
W_{n}=\left\{\xi=\left\{\xi_{k}\right\}_{k \in \mathrm{N}_{0}} \in \Lambda\left(\beta^{\phi}\right): q_{n}(\xi)=\sup _{k}\left(\phi^{-1}\left(\frac{1}{\sqrt{k+1}}\right)\right)^{-n}\left|\xi_{k}\right| \leq 1\right\}
$$

$n \in \mathrm{N}_{0}[10,6.1 .3]$. To prove the continuity of $K_{i}$ we have to find for each neigh bourhood $W_{n}$ a neighbourhood $V_{n} \in \mathcal{U}(E)$ such that $K_{i}\left(V_{n}\right) \subset W_{n}$. Since $G_{n}^{i}$ is equicontinuous we find a neighbourhood $V_{n} \in \mathcal{U}(E)$ with $G_{n}^{i} \subset V_{n}^{0}$ such that for each $x \in V_{n}$

$$
\begin{aligned}
& q_{n}\left(\left\{a_{k}^{i}(x)\right\}_{k \in \mathrm{N}_{0}}\right)=\sup _{k}\left\{\phi^{-1}\left(\frac{1}{\sqrt{k+1}}\right)^{-n}\left|a_{k}^{i}(x)\right|\right\} \\
& \quad \leq \sup \left\{|a(x)|: a \in G_{n}^{i}\right\} \leq \sup \left\{|b(x)|: b \in V_{n}^{0}\right\}=p_{V_{n}}(x) \leq 1 .
\end{aligned}
$$

Thus $K_{i}$ is continuous for each $i \in I$. We are not considering the map $\tilde{K}_{i}: E\left(U_{i}\right)$ $\rightarrow \Lambda\left(\beta^{\phi}\right)$ and its dual $\tilde{K}_{i}^{\prime}:\left[\Lambda\left(\beta^{\phi}\right)\right]_{b}^{\prime} \rightarrow E^{\prime}\left(U_{i}^{0}\right)$ and prove that $U_{i}^{0}$ is the image of an equicontinuous set in $\left[\Lambda\left(\beta^{\phi}\right)\right]_{b}^{\prime}$. Since

$$
\tilde{K}_{i}^{\prime}\left(\left\{\eta_{k}\right\}_{k \in \mathrm{N}_{0}}\right)=\sum_{k=0}^{\infty} \eta_{k} a_{k}^{i}=\sum_{k=0}^{\infty} \eta_{k} \rho(k+1) e_{k}
$$

for $\left\{\eta_{k}\right\}_{k \in N_{0}} \in\left[\Lambda\left(\beta^{\phi}\right)\right]_{b}^{\prime}, U_{i}^{0}$ is the image of the set

$$
B:=\left\{\left\{\rho^{-1}(k+1)^{-1} \eta_{k}\right\}_{k \in \mathrm{N}_{0}}: \sum_{k=0}^{\infty}\left|\eta_{k}\right|^{2} \leq 1\right\} \text {. }
$$

Putting $W:=\left\{\left\{\xi_{k}\right\}_{k \in N_{0}}: \sup _{k}\left|\xi_{k}\right| \leq 1\right\}$ we have for each sequence $\left\{\xi_{k}\right\}_{k \in N_{0}} \in W$

$$
\begin{aligned}
\rho^{-1} \sum_{k=0}^{\infty}(k+1)^{-1}\left|\eta_{k}\right|\left|\xi_{k}\right| & \leq \rho^{-1}\left(\sum_{k=0}^{\infty}\left|\eta_{k}\right|^{2}\right)^{1 / 2}\left(\sum_{k=0}^{\infty} \frac{\left|\xi_{k}\right|^{2}}{(k+1)^{2}}\right)^{1 / 2} \\
& \leq \rho^{-1}\left(\sum_{k=0}^{\infty} \frac{1}{(k+1)^{2}}\right)^{1 / 2} \sup _{k}\left|\xi_{k}\right| \leq 1
\end{aligned}
$$

i.e. $B \subset W^{0}$, thus $B$ is equicontinuous. Therefore the maps $\widetilde{K}_{i}: E\left(U_{i}\right) \rightarrow \Lambda\left(\beta^{\phi}\right)$ and $\Pi_{i \in I} K_{i}: \Pi_{i \in I} E\left(U_{i}\right) \rightarrow\left[\Lambda\left(\beta^{\phi}\right)\right]^{I}$ are open. Since the canonical imbedding $E \rightarrow \prod_{i \in I} E\left(U_{i}\right)$ is a (topological) isomorphism, the imbedding $E \rightarrow\left[\Lambda\left(\beta^{\phi}\right)\right]^{I}$ is a (topological) isomorphism, too.

Corollary. If for $\phi \in \Phi$ the power series space $\Lambda\left(\beta^{\phi}\right)$ is $\phi$-nuclear then $\Lambda\left(\beta^{\phi}\right)$ is a universal generator for the variety $\pi_{\phi}$ of $\phi$-nuclear spaces. 
The corollary to Theorem 4.7 describes a universal generator for the variety $r_{\phi}$ in case $\Lambda\left(\beta^{\phi}\right)$ is $\phi$-nuclear. It is therefore natural to ask for a concrete universal generator for the variety $\gamma_{\phi}$ if $\Lambda\left(\beta^{\phi}\right)$ is not $\phi$-nuclear.

Martineau [8] states that $(s)^{\prime}$ is a universal generator for the variety $r_{s}$ of all $s$ nuclear spaces where $s$ is the space of rapidly decreasing sequences. Dubinsky and Ramanujan [4] generalized this result by proving that the class $r_{\Lambda(a)}-\Lambda(a)$ a nuclear power series space - is a variety if and only if the sequence $\alpha=\left\{a_{n}\right\}_{n} \in N_{0}$ satisfies the condition $\sup _{n} a_{n}^{-1} \alpha_{2 n}<\infty$. In this case the universal generator of $\pi_{\Lambda(a)}$ is $[\Lambda(\alpha)]_{b}^{\prime}$. So one might think of $\left[\Lambda\left(\beta^{\phi}\right)\right]_{b}^{\prime}$ being a universal generator for the variety $r_{\phi}$ if $\Lambda\left(\beta^{\phi}\right)$ is not $\phi$-nuclear. But the following example shows that $\left[\Lambda\left(\beta^{\phi}\right)\right]_{b}^{\prime}$ does not have to be $\phi$-nuclear if $\Lambda\left(\beta^{\phi}\right)$ is not $\phi$-nuclear.

Example. Take $\phi=\phi_{\text {log }}$. By Lemma 4.5 (iii), $\Lambda\left(\beta^{\phi}\right)$ is not $\phi$-nuclear. But since $\Lambda\left(\beta^{\phi}\right)$ is nuclear (Lemma 4.5 (ii)), we have that $\left[\Lambda\left(\beta^{\phi}\right)\right]_{b}^{\prime}=\Lambda^{\prime}(Q)$ where $Q=\left\{\xi=\left\{\xi_{k}\right\}_{k \in N_{0}} \in \Lambda\left(\beta^{\phi}\right): \xi_{k} \geq 0\right\}$ and $\Lambda^{\prime}(Q)$ has its normal topology [6]. Suppose $[\Lambda(\beta \phi)]_{b}^{\rho}$ is $\phi$-nuclear; we may find for each sequence $\left\{\xi_{k}\right\}_{k \in N_{0}} \in \Lambda\left(\beta^{\phi}\right)$ with $\xi_{k} \geq 0$ a sequence $\left\{\eta_{k}\right\}_{k \in N_{0}} \in \Lambda\left(\beta^{\phi}\right)$ with $\eta_{k} \geq 0$ and a sequence $\left\{\mu_{k}\right\}_{k \in N_{0}} \in l_{\phi}^{+}$such that $\xi_{k} \leq \eta_{k} \mu_{k}$ for each $k_{\phi} \in N_{0}$ [13, Satz 3.3]. Thus $\Lambda(\beta \phi) \subset l_{\phi}$. Since $\Lambda\left(\beta^{\phi}\right)=\left\{\left\{\xi_{k}\right\}_{k \in N_{0}}:\left|\xi_{k}\right|^{\left.1 / \beta_{k}^{\phi} \rightarrow 0\right\} \text { we have }}\right.$

$$
\left\{\xi_{k}\right\}_{k \in N_{0}} \in \Lambda\left(\beta^{\phi}\right) \Leftrightarrow \frac{-\log \left(\phi^{-1}(1 / \sqrt{k+1})\right)}{-\log \left|\xi_{k}\right|} \rightarrow 0 \Leftrightarrow \sqrt{k+1} \phi_{\log }\left(\xi_{k}\right) \rightarrow 0
$$

Therefore it is easily seen that $\Lambda\left(\beta^{\phi}\right) \not \subset l_{\phi}$, and $\left[\Lambda\left(\beta^{\phi}\right)\right]_{b}^{\prime}$ is not $\phi$-nuclear.

For a certain class of functions $\phi \epsilon \Phi$ we give a result similar to the result of Dubinsky and Ramanujan.

Lemma 4.8. For $l_{\phi}^{+}:=\left\{\xi=\left\{\xi_{k}\right\}_{k \in N_{0}} \in l_{\phi}: \xi_{k} \geq 0\right\}$ the following assertions are equivalent:

(i) The sequence space $\Lambda\left(l_{\phi}^{+}\right)$is $\phi$-nuclear, $\phi \in \Phi$.

(ii) $\lim \sup _{t \rightarrow 0}(\phi(t))^{-1} \phi(\sqrt{t})<\infty$.

(iii) For each $p>0$, lim $\sup _{t \rightarrow 0}(\phi(t))^{-1} \phi\left(t^{p}\right)<\infty$.

Proof. (i) $\Rightarrow$ (ii). Suppose lim sup $\operatorname{sio}_{t \rightarrow}(\phi(t))^{-1} \phi(\sqrt{t})=\infty$; then by Lemma 3.1. $l_{\phi} \not t$ $l_{\phi \circ \phi 1 / 2}$ where $\phi \circ \phi_{1 / 2} \in \Phi$ with $\phi \circ \phi_{1 / 2}(t)=\phi(\sqrt{t})$. On the other hand, since $\Lambda\left(l_{\phi}^{+}\right)$is $\phi$-nuclear, we have that for each sequence $\xi \in l_{\phi}^{+}$there exists a sequence $\eta \in l_{\phi}^{+}$ and a sequence $\mu \in l_{\phi}^{+}$such that $\xi_{k} \leq \eta_{k} \mu_{k}$ for $k \in \mathbf{N}_{0}[13$, Satz 3.3]. By the Hölder inequality (Theorem 3.3) we therefore have $l_{\phi}=l_{\phi \circ \phi 1 / 2} \cdot$

(ii) $\Rightarrow$ (iii). It is easily seen that (iii) is true for $p \geq 1 / 2$. So let be $0<p<1 / 2$. We may choose $m \in \mathbf{N}$ such that $p 2^{m}>1$. From

$$
\frac{\phi\left(t^{1 / 2^{m}}\right)}{\phi(t)}=\prod_{k=1}^{m} \frac{\phi\left(t^{1 / 2^{k}}\right)}{\phi\left(t^{1 / 2^{k-1}}\right)}
$$


it easily follows that $\lim \sup _{t \rightarrow 0}(\phi(t))^{-1} \phi\left(t^{1 / 2^{m}}\right)<\infty$ and therefore $\lim \sup _{t \rightarrow 0}(\phi(t))^{-1} \phi\left(t^{p}\right)<\infty$.

(iii) $\Rightarrow$ (ii). Trivial.

(ii) $\Rightarrow$ (i) is a consequence of Lemma 3.1 and [13, Satz 3.3].

In the sequel we denote by $\Phi_{w}$ the class of functions $\phi \in \Phi$ having property (ii) in Lemma 4.8.

Remark. If $\Lambda\left(\beta^{\phi}\right)$ is $\phi$-nuclear then, by Lemma 4.5 and Lemma $4.8, \phi \notin \Phi_{W}$.

Theorem 4.9. For $\phi \in \Phi_{W}$ the sequence space $\Lambda\left(l_{\phi}^{+}\right)$is a universal generator for the variety $\hat{\gamma}_{\phi}$ of $\phi$-nuclear spaces.

Proof. By Lemma 4.8 the sequence space $\Lambda\left(l_{\phi}^{+}\right)$is $\phi$-nuclear and therefore by $\left[13\right.$, Satz 2.5] arbitrary products of $\Lambda\left(l_{\phi}^{+}\right)$are $\phi$-nuclear.

Suppose now that $E$ is a $\phi$-nuclear space. Let $\mathcal{U}(E)=\left\{U_{i}\right\}_{i \in l}$ be a fundamental system of (closed, absolutely convex, and absorbing) neighbourhoods of 0 where $I$ is a suitably determined index set. Since $E$ is $\phi$-nuclear, by Theorem 3.4 we can find to each $U_{i} \in \mathcal{U}(E)$ a $V_{i} \in \mathcal{I}(E)$ with $V_{i}<U_{i}$ such that the canonical map $\widehat{E\left(V_{i}, U_{i}\right)}$ of $\widehat{E\left(V_{i}\right)}$ into $\widehat{E\left(U_{i}\right)}$ is $\phi$-nuclear and therefore admits the representation

$$
x\left(U_{i}\right)=\widehat{E\left(V_{i}, U_{i}\right)} x\left(V_{i}\right)=\sum_{k=1}^{\infty} \lambda_{k}^{(i)} a_{k}^{(i)}\left(x\left(V_{i}\right)\right) y_{k}^{(i)}
$$

for each $x\left(V_{i}\right) \in \widehat{E\left(V_{i}\right)}$ where $\left\{\lambda_{k}^{(i)}\right\}_{k \in N} \in l_{\phi}, a_{k}^{(i)} \in \widehat{\left[E\left(V_{i}\right)\right]_{b}^{\prime}},\left\|a_{k}^{(i)}\right\| \leq 1$, and $y_{k}^{(i)} \in \widehat{E\left(U_{i}\right)}$ with $\left\|y_{k}^{(i)}\right\| \leq 1$ for each $k \in \mathbb{N}$. Thus we can get $b_{k}^{(i)} \in \bar{V}_{i}^{0}$ for each $k \in \mathbf{N}$, so that

$$
p_{U_{i}}(x) \leq \sum_{k=1}^{\infty}\left|\lambda_{k}^{(i)}\right|\left|b_{k}^{(i)}\left(x\left(V_{i}\right)\right)\right| \leq p_{V_{i}}(x) \sum_{k=1}^{\infty}\left|\lambda_{k}^{(i)}\right| \text {. }
$$

For $i \in I$ let $K_{i}: E \rightarrow \Lambda\left(l_{\phi}^{+}\right)$be defined by $K_{i}(x)=\left\{b_{k}^{(i)}(x)\right\}_{k \in N}$. Since for each sequence $\left\{\delta_{k}\right\}_{k \in N} \in l_{\phi}^{+}$we have

$$
\sum_{k=1}^{\infty}\left|b_{k}^{(i)}(x)\right| \delta_{k} \leq p_{V_{i}}(x) \sum_{k=1}^{\infty} \delta_{k} \leq p_{V_{i}}(x) \phi^{-1}\left(\sum_{k=1}^{\infty} \phi\left(\delta_{k}\right)\right),
$$

it follows that $K_{i}(x)$ actually belongs to $\Lambda\left(l_{\phi}^{+}\right)$for each $x \in E$. Now let $K: E \rightarrow$ $\Pi_{i \in I} \Lambda\left(l_{\phi}^{+}\right)=\left[\Lambda\left(l_{\phi}^{+}\right)\right]^{l}$ defined by $K x=\left\{K_{i}(x)\right\}_{i \in I}$. Since $K_{i}$ and $K$ are linear, it follows from $(*)$ that $K$ is a one-to-one map. We are now proving that $K_{i}$ is continuous for each $i \in I$. For an arbitrary sequence $\left\{\delta_{k}\right\}_{k \in \mathbb{N}} \in l_{\phi}$ or equivalently, an arbitrary seminorm $p_{\delta}$ generating the topology of $\Lambda\left(l_{\phi}^{+}\right)$, we have $\sum_{k=1}^{\infty}\left|b_{k}^{(i)}(x)\right| \delta_{k}$ $\leq p_{V_{i}}(x) \delta_{0}$ for each $i \in I$ where $\delta_{0}:=\Sigma_{k=1}^{\infty} \delta_{k}$. Therefore the linear map $K_{i}$ is continuous. Since the projection $P_{i}$ of $\left[\Lambda\left(l_{\phi}^{+}\right)\right]^{l}$ onto $\Lambda\left(l_{\phi}^{+}\right)$is continuous and since $K_{i}=P_{i} \circ K$ we get that $K$ is continuous. To show that $K^{-1}$ is continuous consider the neighbourhood 


$$
W_{i}:=\left\{\xi=\left\{\xi_{k}\right\}_{k \in N} \in \Lambda\left(l_{\phi}^{+}\right): \sum_{k=1}^{\infty}\left|\xi_{k}\right|\left|\lambda_{k}^{(i)}\right| \leq 1\right\} .
$$

Then $P_{i}^{-1}\left(W_{i}\right)=\hat{W}_{i}$ is a neighbourhood in the product space $\left[\Lambda\left(l_{\phi}^{+}\right)\right]^{l}$. Using $(*)$ it is easily seen that $K^{-1}\left(\hat{W}_{i}\right) \cap E \subset U_{i}$, i.e., $K^{-1}$ is continuous.

As an immediate consequence of this theorem we have

Corollary. The sequence space $\Lambda\left(l_{\phi_{\text {log }}}^{+}\right)$is a universal generator for the variety $r_{\phi_{\mathrm{log}}}$ of $\phi_{\mathrm{log}}$-nuclear spaces where $\phi_{\mathrm{log}} \in \dot{\Phi}$ is given in 2.6 (Example (ii)).

The following theorem gives an idea how functions $\phi \in \Phi_{W}$ can be generated by a given function $\tilde{\phi} \in \Phi_{W}$.

Theorem 4.10. Let $\phi \in \Phi_{W}$. If for a function $\psi \in \Phi,(\psi(t))^{-1} \phi(t)$ is increas. ing in $(0, \infty)$, then $\psi \in \Phi$ satisfies condition (ii) of Lemma 4.8, i.e. $\psi \in \Phi_{W}$.

Proof. Since $\phi \in \Phi_{W}$, the inverse function $\phi^{-1}$ exists, is continuous and strictly increasing in $[0, \infty)$ with $\phi^{-1}(0)=0 \cdot t^{-1} \psi\left(\phi^{-1}(t)\right)$ is decreasing in $(0, \infty)$ because $(\psi(t))^{-1} \phi(t)$ is increasing; therefore

$$
\begin{aligned}
\psi\left(\phi^{-1}(s+t)\right) & =s \frac{\psi\left(\phi^{-1}(s+t)\right)}{s+t}+t \frac{\psi\left(\phi^{-1}(s+t)\right)}{s+t} \\
& \leq s \frac{\psi\left(\phi^{-1}(s)\right)}{s}+t \frac{\psi\left(\phi^{-1}(t)\right)}{t}=\psi\left(\phi^{-1}(s)\right)+\psi\left(\phi^{-1}(t)\right),
\end{aligned}
$$

for $s, t \in(0, \infty)$. Thus $\psi \circ \phi^{-1}$ is subadditive, and there exists a positive number $\epsilon$ and $M \in \mathbf{N}$ such that

$$
\psi(\sqrt{t})=\psi \circ \phi^{-1} \circ \phi(\sqrt{t}) \leq \psi \circ \phi^{-1}(M \phi(t)) \leq M \psi(t)
$$

for $0 \leq t<\epsilon$, i.e. $\psi \in \Phi_{W}$.

The following example shows that in addition to the trivial cases $\phi_{p} \in \Phi$, $0<p \leq 1$, there are functions $\phi \in \Phi$ such that $\phi \notin \Phi_{W}$.

Example. Take the example (iii) in 2.6.

$$
\psi^{q}(t)= \begin{cases}0 & \text { for } t=0, \\ a_{q} \exp \left(-(\log 1 / t)^{q}\right) & \text { for } t \in\left(0, t_{0}\right], \\ b_{q} t+c_{q} & \text { for } t \geq t_{0},\end{cases}
$$

with $0<q \leq 1, t_{0}$ sufficiently small and $a_{q^{\prime}} b_{q^{0}} c_{q}$ properly chosen. It is easily seen that

$$
\begin{aligned}
\lim _{t \rightarrow 0}\left(\psi^{q}(t)\right)^{-1} t^{p} & =\lim _{t \rightarrow 0, t \leq t_{0}} a_{q}^{-1} \exp \left(-p \log \frac{1}{t}+\left(\log \frac{1}{t}\right)^{q}\right) \\
& =\lim _{s \rightarrow \infty} \exp \left(s^{q}-p s\right)=0 .
\end{aligned}
$$


On the other hand we have for each $0<q \leq 1$

$$
\begin{aligned}
\lim _{t \rightarrow 0}\left(\psi^{q}(t)\right)^{-1} \psi^{q}(\sqrt{t}) & =\lim _{t \rightarrow 0, t \leq t_{0}} \exp \left(-\left(\log \frac{1}{\sqrt{t}}\right)^{q}+\left(\log \frac{1}{t}\right)^{q}\right) \\
& =\lim _{t \rightarrow 0, t \leq t_{0}}\left(\exp \left(\frac{2^{q}-1}{2^{q}}\right)\right)^{(\log 1 / t)^{q}} \\
& =\infty
\end{aligned}
$$

i.e. $\psi^{q} \notin \Phi_{W}, 0<q \leq 1$.

The corresponding power series spaces $\Lambda\left(\beta^{\psi^{q}}\right)$ are $\psi^{q}$-nuclear for $0<q \leq 1$ since

for $m>2$.

$$
\sum_{k \geq k_{0}} \psi_{q}\left(\left(\psi_{q}^{-1}\left(\frac{1}{\sqrt{k+1}}\right)\right)^{m}\right)=\sum_{k \geq k_{0}}(k+1)^{-(m / 2)^{q}}<\infty
$$

By the remark following Lemma 4.8 the $\phi$-nuclearity of $\Lambda\left(\beta^{\phi}\right)$ implies $\phi \notin \Phi_{W}$, but on the other hand there are functions $\phi \in \Phi$ with $\phi \notin \Phi_{W}$ such that $\Lambda\left(\beta^{\phi}\right)$ is not $\phi$-nuclear.

Example.(1) Let $\phi:[0, \infty) \rightarrow[0, \infty)$ be defined as follows:

$$
\phi(t):= \begin{cases}a t+b & \text { for } t \geq t_{0}, t_{0} \text { sufficiently small, } \\ a_{k}(\log 1 / t)^{-k} & \text { for } t \in\left[1 / \tau_{k+1}, 1 / \tau_{k}\right], k \in \mathrm{N},\end{cases}
$$

where $t_{0}=r_{1}^{-1}, a, b$ are properly chosen and $r_{k}, k \in \mathrm{N}$, are chosen in such a way that $r_{k+1}>r_{k}$ with

$$
\sum_{i=\tau}^{r+1} i_{k}^{-1} \geq k^{k}, \quad k \in \mathrm{N} \text {. }
$$

The positive numbers $a_{k}$ are chosen in such a way that $a_{k}\left(\log r_{k+1}\right)^{-k}=$ $a_{k+1}\left(\log \tau_{k+1}\right)^{-k-1}$, i.e. $a_{k+1}=a_{k} \log \tau_{k+1}$, where $a_{1}=1$.

(i) It is easily seen that the function $\phi$ is continuous in $(0, \infty)$ and since

$$
a_{k}\left(\log \tau_{k+1}\right)^{-k}=\left(\log \tau_{k+1}\right)^{-k} \prod_{i=2}^{k} \log \tau_{i} \leq\left(\log \tau_{k+1}\right)^{-1} \text {, }
$$

$k \in \mathbf{N}$, we may put $\phi(0)=0$. The function $\phi$ is obviously strictly increasing. Thus by 2.6 it remains to prove that $\phi$ is subadditive. For $s, t \in\left(0, t_{0}\right]$ we may assume that $s \in\left[r_{k+i+1}^{-1}, \tau_{k+i}^{-1}\right]$ and $t \in\left[\tau_{k+1}^{-1}, r_{k}^{-1}\right]$ for $k \in N, i \in\{2,3, \ldots\}$, since for $s, t \in\left[\tau_{k+1}^{-1}, r_{k}^{-1}\right]$ (resp. $s \in\left[r_{k+2}^{-1}, r_{k+1}^{-1}\right], t \in\left[r_{k+1}^{-1}, r_{k}^{-1}\right]$ ) it is obvious that $t^{-1} \phi(t) \leq s^{-1} \phi(s)$. We then have

(1) This example was given by Professor S. Rolewicz in a private communication. 


$$
\begin{aligned}
t^{-1} \phi(t) & \leq \phi\left(\tau_{k+1}^{-1}\right) \tau_{k+1}=\tau_{k+1} a_{k}\left(\log \tau_{k+1}\right)^{-k}=\tau_{k+1} a_{k+1}\left(\log \tau_{k+1}\right)^{-k-1} \\
& \leq \tau_{k+2} a_{k+2}\left(\log \tau_{k+2}\right)^{-k-1}=\tau_{k+2} a_{k+2}\left(\log \tau_{k+2}\right)^{-k-2} \\
& \leq \cdots \leq \tau_{k+i} a_{k+i}\left(\log \tau_{k+i}\right)^{-k-i} \leq s^{-1} \phi(s) .
\end{aligned}
$$

Thus $t^{-1} \phi(t)$ is strictly decreasing in $\left(0, t_{0}\right]$ and by the same argument as in Theorem 4.10. we can show that $\phi$ is subadditive.

(ii) Since in $\left[\tau_{k+1}^{-1}, \tau_{k}^{-1}\right]$ the inverse function of $a_{k}(\log 1 / t)^{-k}$ is $\exp \left(-\left(t^{-1} a_{k}\right)^{1 / k}\right)$, for each $m \in N$ we have

$$
\begin{aligned}
\sum_{k=1}^{\infty} \phi\left(\left(\phi^{-1}\left(\frac{1}{k+1}\right)\right)^{m}\right) & \geq \sum_{k=1}^{\infty} \sum_{i=\tau}^{\tau+1} \phi\left(\left(\phi^{-1}\left(\frac{1}{i}\right)\right)^{m}\right) \\
& =\sum_{k=1}^{\infty} m^{-k} \sum_{i=\tau}^{r+1} \frac{1}{i} \geq \sum_{k=1}^{\infty}\left(\frac{k}{m}\right)^{k}=\infty
\end{aligned}
$$

Thus by Lemma 4.5 the corresponding power series space $\Lambda\left(\beta^{\phi}\right)$ is not $\phi$-nuclear.

(iii) But on the other hand for $t \in\left[\tau_{k+1}^{-1}, \tau_{k}^{-1}\right]$ we have

$$
(\phi(t))^{-1} \phi(\sqrt{t})=2^{k} a_{k}(\log 1 / t)^{-k} a_{k}^{-1}(\log 1 / t)^{k}=2^{k}
$$

and therefore $\lim _{t \rightarrow 0}(\phi(t))^{-1} \phi(\sqrt{t})=\infty$, i.e. $\phi \notin \Phi_{W^{*}}$

\section{REFERENCES}

1. C. Bessaga, A. Pelczyński and S. Rolewicz, Some properties of the norm of $F$ spaces, Studia Math. 16 (1957), 183-192. MR 20 \#6021.

2. - On diametral approximative dimension and linear homogeneity of F-spaces, Bull. Acad. Polon. Sci. Sér. Sci. Math. Astronom. Phys. 9 (1961), 677-683. MR 24 \#A2219.

3. J. Diestel, S. A. Morris and S. A. Saxon, Varieties of linear topological spaces, Trans. Amer. Math. Soc. (to appear).

4. E. Dubinsky and M. S. Ramanujan, On $\lambda$-nuclearity, Mem. Amer. Math. Soc. No. 128 (1972).

5. G. Köthe, Topologische lineare Räume. I, 2nd ed., Die Grundlehren der math. Wissenschaften, Band 107, Springer-Verlag, Berlin, 1966; English transl., Die Grundlehren der math. Wissenschaften, Band 159, Springer-Verlag, New York, 1969. MR 33 \#3069; $40 \# 1750$.

6. — Über nukleare Folgenräume, Studía Math. 31 (1968), 267-271. MR 38 \#4946.

7. T. Kōmura and Y. Kōmura, Über die Einbettung der nuklearen Räume in $(s)^{A}$, Math. Ann. 162 (1965/66), 284-288. MR 32 \#6190.

8. A. Martineau, Sur une properiété universelle de l'espace des distributions de M. Schwartz, C. R. Acad. Sci. Paris 259 (1964), 3162-3164. MR 29 \#6306.

9. S. Mazur and W. Orlicz, On some clas ses of linear spaces, Studia Math. 17 (1958), 97-119. MR 20 \#4780. 
10. A. Pietsch, Nukleare lokalkonvexe Räume, Akademie-Verlag, Berlin, 1965; Russian transl., Mir, Moscow, 1967. MR 31 \#6114; 36 \#4314.

11. M. S. Ramanujan, Power series spaces $\mathrm{\Lambda}(a)$ and associated $\mathrm{\Lambda}(a)-$ nuclearlity, Math. Ann. 189 (1970), 161-168. MR 42 \#5003.

12. B. Rosenberger, F-Normideale von Operatoren in normierten Räumen, Berichte Ges. Math. Daten v. Bonn 44 (1971), 1-40.

13. -

14. B. Rosenberger and E. Schock, Über nukleare (F)-Räume mit Basis, Compositio Math. 25 (1972), 207-219.

INSTITUT FÜR ANGEWANDTE MATHEMATIK DER UNIVERSITÄT BONN, D 5300 BONN, FEDERAL REPUBLIC OF GERMANY 